\title{
Accurate left ventricular segmentation based on morphological watershed transformation towards 3D visualization
}

\author{
KhouloudBOUKHRIS ${ }^{1[0000-0001-6187-720 X]}$, Ramzi MAHMOUDI 1,2[0000-0002-4271-3506], \\ Badii HMIDA $^{3}$ [0000-0003-3109-1331], Mohamed Hédi BEDOUI ${ }^{1}$ [0000-0003-4846-1722] \\ ${ }^{1}$ Faculty of Medicine of Monastir, Medical Imaging Technology Lab - LTIM-LR12ES06, \\ University of Monastir - 5019 MONASTIR, TUNISIA. \\ ${ }^{2}$ Gaspard-Monge computer-science laboratory, Paris-Est University, Mixed Unit CNRS- \\ UMLV-ESIEE UMR8049, BP99, ESIEE Paris City Descartes, 93162 Noisy Le Grand, \\ FRANCE. \\ ${ }^{3}$ Radiology Service- UR12SP40 CHU Fattouma Bourguiba - 5019 MONASTIR, TUNISIA. \\ boukhris. khouloudegmail.com
}

\begin{abstract}
The current challenge for image treatment, targeting the heart ventricles (LV and RV), consists on ensuring an accurate automatic segmentation aiming to identify the myocardial contours. In this paper we propose an automatic watershed-based segmentation of the left ventricle (LV)based on topology, geometry and brightness priors. We attempted to demonstrate the accuracy of our proposed approach by comparing it with other manual segmentations carried out by two clinical experts, on 20 patients. In the aim of obtaining an accurate $3 \mathrm{D}$ reconstruction, we propose the volumetric object reconstruction method using The Visualization Toolkit VTK .Furthermore, the 3D+t assessment is used for the examination of the dynamic behavior of the LV to reveal regions of myocardial dysfunction.
\end{abstract}

Keywords: Watershed, Segmentation, Left Ventricular, Accuracy, 3D.

\section{Introduction}

Cardiac magnetic resonance imaging (cMRI) represents the golden reference method for assessing the LV function and analyzing the myocardial diseases [1], using mainly short-axis cine images. With the aim of obtaining full exploitation of the information contained in these MRI images, the automated extraction and segmentation of the myocardium contours, which are often drawn manually by cardiologists, are proving to be crucial [2].In this paper, we propose to compare a set of existing methods dealing with ventricular segmentation in short axis cine MR images, in order to prove the accuracy of our proposed method. This comparison was also assessed against manual segmentations performed by cardiologist. The main issue with an accurate LV segmentation is to ensure its faithful 3-D reconstruction. This paper is organized as follows: section 2 describes the previous work aiming to segment the heart cavities. 
Section 3 and 4 are dedicated to introduce then evaluate the proposed segmentation process to extract the LV myocardium. Section 5 depicts resulting 3D visualization. Finally, section 6 presents the conclusion.

\section{Review of ventricular segmentation methods in cMRI}

Cardiac Magnetic Resonance Imaging (cMRI) is used to help visualizing the internal structures of the human heart in order to highlight the medical management of patients. MRI cardiac planes include short axis, horizontal long axis (four-chamber view), and vertical long axis (two-chamber view) [3]. Various segmentation methods have been proposed by different researchers using generally the short-axis view [4]. These existing methods can be divided into two main categories: "segmentation based on no or weak prior" and "segmentation based on a strong prior". Approaches based on no or weak prior are very contingent upon the gray-level histograms and the thresholding techniques. These approaches include image-based methods [5,6,7], pixel classification and deformable models [8, 9]. However, approaches based on strong prior are characterized by the use of statistical models which are mainly divided into three categories: deformable models based segmentation with strong prior, Active shape ("ASM") [10, 11] and active appearance models ("AAM") [12] and atlas based ones [13, 9, 14].

\section{Proposed method based on watershed transform}

Since the early work of Serge Beucher[15], the watershed transform has been successfully applied in thousands of applications, including medical ones. Our approach is based mainly on the watershed transformation method [16] and considers prior properties. The set of prior knowledge that will allow an accurate segmentation are: topology (the left ventricular myocardium LVM is delimited by two surfaces: endocardium and epicardium), geometry (the endocardium cannot exceed the epicardium) and brightness (the endocardium is a very bright object). Our proposed method to automatically segment the LVM in 3D+t short-axes images is composed of two consecutive steps: endocardium segmentation, followed by the epicardium segmentation. Thereafter, the myocardium extraction (LVM) consists in applying the ensemblist difference between the epicardial and the endocardial civility. The segmentation of the endocardium is performed by geodesic dilations. Its recognition is ensured by extracting a connected set of pixels that belongs to the endocardium (thanks to its brightness). The delineation is then performed by dilating this marker in a mask made to be a candidate that belongs to the endocardium. The epicardium segmentation is consequent to the endocardium one preserving certain anatomical constraints. We propose to separate the myocardial cavity from the ventricular background. To achieve this goal, we propose to dilate the endocardium contour in order to obtain the first epicardial marker (EPm).To extract the EPm, the idea is to dilate the endocardi- 
um segmented cavity (ENC) as much as possible by ensuring that the resulting set is included in the real ENC, according to the prior:

$$
\text { Epicardium_Boundary } \cap \text { Endocardium_Boundary }=\varnothing
$$

The next step consists of finding a marker for the left ventricular background (LVBm) that does not belong to the myocardium: composed of several tissues including the liver, the stomach, the right ventricle and the lungs. In order to extract the LV background marker (LVBm), we propose a second dilatation, based on the prior that the myocardium thickness cannot exceed a certain threshold. Then, the delineation is performed by the topological watershed transformation approach cited in [16] using the above-selected markers as inputs. The delineation is followed by a smoothing post-processing using shape filters coming from mathematical morphology. Thus, in order to obtain the final segmentation, we use the alternating sequential filter (ASF) [18] to regularize the epicardial boundary (Ep) and to restore a correct shape of the intensity information. The ASF is a sequence of intermixed morphological openings and closings by balls of an increasing size.

\section{$4 \quad$ Validation of the proposed segmentation}

In our experiment, we used 4D cine MR images of 20 patients to segment. For the evaluation, the same dataset was manually segmented by two different experts, called belowExp1 and Exp2.

\subsection{Point to surface measurement}

Given two surfaces $\mathrm{S}$ and $\mathrm{S}$ ' represented by two polygons, the point-to-surface measurement (P2S) defined in [19] estimates the mean distance between the vertices of these surfaces ( $\mathrm{S}$ and $\mathrm{S}$ ').

Table 1.Literature values of left ventricle delineation errors.

\begin{tabular}{lcc}
\hline & \multicolumn{2}{c}{ Distance (mm) } \\
\hline & EndoS & EpiS \\
\hline Mitchell and al. [11] & 2.75 & 2.63 \\
Kaus and al. [20] & 2.28 & 2.62 \\
Lötjönen and al. [14] & 2.01 & 2.77 \\
Van Assen and al. [12] & 1.97 & 2.23 \\
Lorenzo-Valdes and al. [9] & 1.88 & 2.75 \\
Jolly and al. [6] & 2.48 & 2.91 \\
Ying-Li Lu and al. [21] & 2.07 & 1.92 \\
Huaifei Hu and al. [22] & 2.3 & 2.2 \\
Irshad and al. [23] & 2.1 & 3.1 \\
Proposed method & 1.52 & 1.75 \\
\hline
\end{tabular}


From our proposed segmentation, the endocardial and the epicardial surfaces (EndoS and EpiS) were used to compute the P2S in order to compare them with those obtained by other groups on their own datasets [6,9,11,12,14,20,21,22,23].In table1, different distance measures similar to P2S where used: APD (Average Perpendicular distance), Hausdroff distance and image volumes. The comparison demonstrates that the distance (P2S) evaluation results averaged favorably across our proposed method and those obtained by previous works. The P2S was computed from the segmentations resulting from our proposed method and the two experts. In order to assess the inter-observer variability, the P2S of both experts is also provided as shown in table 2.

Table 2.Results of the P2S(mm) measurements from our proposed method (PM).

\begin{tabular}{clccc}
\hline & & PM VS. EXP1 & PM VS.EXP2 & EXP1 VS.EXP2 \\
\hline $\begin{array}{c}\text { End- } \\
\text { diastolic time }\end{array}$ & EndoS & 1.62 & 1.52 & 1.46 \\
& EpiS & 1.81 & 1.60 & 1.62 \\
$\begin{array}{c}\text { End-systolic } \\
\text { time }\end{array}$ & EndoS & 1.45 & & \\
\hline
\end{tabular}

The obtained results are convergent (PM vs.exp and exp vs. exp) with a distance that does not exceed $2 \mathrm{~mm}$, thus evidencing the reliability of the proposed segmentation.

\subsection{False negative and false positive Volume Fraction}

These two measures are recommended by [19] to characterize the delineation accuracy. Considering Struth the set of image pixels containing 'true' delineations of the $\mathrm{LV}$ and Spm the segmentation to be compared to the ground truth to be evaluated, we set False Negative Ejection Fraction FNEF and False Positive Ejection Fraction FPEF:

$$
\begin{aligned}
& F P V F(\text { Spm,Struth })=\frac{\mid \text { Spm } \backslash \text { Struth } \mid}{\text { Struth }} \\
& F N V F(\text { Spm,Struth })=\frac{\mid \text { Struth } \backslash \text { Spm } \mid}{\text { Struth }}
\end{aligned}
$$

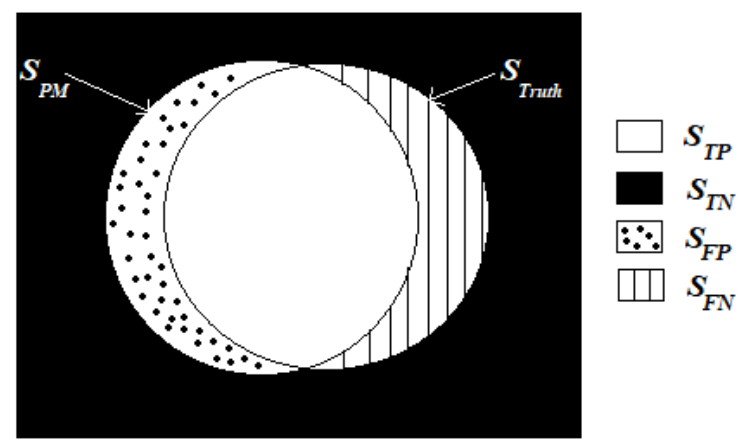

Fig. 1.Illustration of the accuracy factors from a binary case segmentation. 
Figure1 illustrates the meaning of these measures: A false positive volume fraction (FPVF)indicates the amount of pixels falsely extracted to be considered as a fraction of the true delineation (considered as the reference segmentation) and the false negative volume fraction (FNVF) depicts the fraction of pixels that was missed. These metrics were computed for the three segmented areas: the epicardium, the endocardium and the myocardium. Table 3 depicts their mean values between the proposed method PM and the two experts at end-systolic and end-diastolic time. In order to achieve an accurate assessment, these measures were also provided between the two experts. The inter-expert segmentations results (exp1 vs.exp2 and exp2 vs. exp1) compare well to those obtained between the proposed method and expert segmentations (PM vs. exp1 and PM vs. exp2). The Dice metric can be expressed in terms of true positives (TP), false positives (FP) and false negatives (FN) as:

$$
\operatorname{Dice}(A, B)=(2 * T P) /(2 * T P+F P+F N)
$$

Comparing our method to both experts, the best segmentation result had a Dice Metric of 0.96 and the worst a DC of 0.73 .

Table 2.FPVF and FNVF values for the segmentation of the endocardium, the epicardium and the myocardium at end-systolic and end-diastolic time.

\begin{tabular}{|c|c|c|c|c|c|}
\hline & & PM vs. exp1 & PM vs.exp2 & Exp1 vs. exp2 & Exp2 vs. exp1 \\
\hline \multicolumn{6}{|c|}{ End-systolic-time } \\
\hline \multirow{2}{*}{ Endocardium } & FPVF & 0.04 & 0.05 & 0.15 & 0.02 \\
\hline & FNVF & 0.12 & 0.07 & 0.03 & 0.14 \\
\hline \multirow{2}{*}{ Epicardium } & FPVF & 0.08 & 0.14 & 0.06 & 0.09 \\
\hline & FNVF & 0.21 & 0.06 & 0.10 & 0.12 \\
\hline \multirow{2}{*}{ Myocardium } & FPVF & 0.02 & 0.10 & 0.25 & 0.09 \\
\hline & FNVF & 0.04 & 0.22 & 0.14 & 0.08 \\
\hline \multicolumn{6}{|c|}{ End-diastolic-time } \\
\hline \multirow{2}{*}{ Endocardium } & FPVF & 0.06 & 0.12 & 0.09 & 0.12 \\
\hline & FNVF & 0.12 & 0.23 & 0.21 & 0.10 \\
\hline \multirow{2}{*}{ Epicardium } & FPVF & 0.09 & 0.11 & 0.09 & 0.06 \\
\hline & FNVF & 0.14 & 0.02 & 0.13 & 0.07 \\
\hline \multirow{2}{*}{ Myocardium } & FPVF & 0.04 & 0.08 & 0.18 & 0.21 \\
\hline & FNVF & 0.03 & 0.13 & 0.09 & 0.04 \\
\hline
\end{tabular}




\section{The 3D visualization}

Searching for a means to display the segmentation results proved to be important. To achieve it, we start by generating meshes from the binary images resulting from the proposed segmentation. The ability to visualize the LV meshes would be a significant advantage. To generate these meshes we applied the method defined in [24] using the marching cube algorithm [25].The ensuing step consists on applying Laplacian smoothing steps [26] on these resulting meshes and save them in the vtk format [27].Figure 2 depicts an example of myocardium segmentation ranging from apical to basal plane and the extracted mesh from different corners.
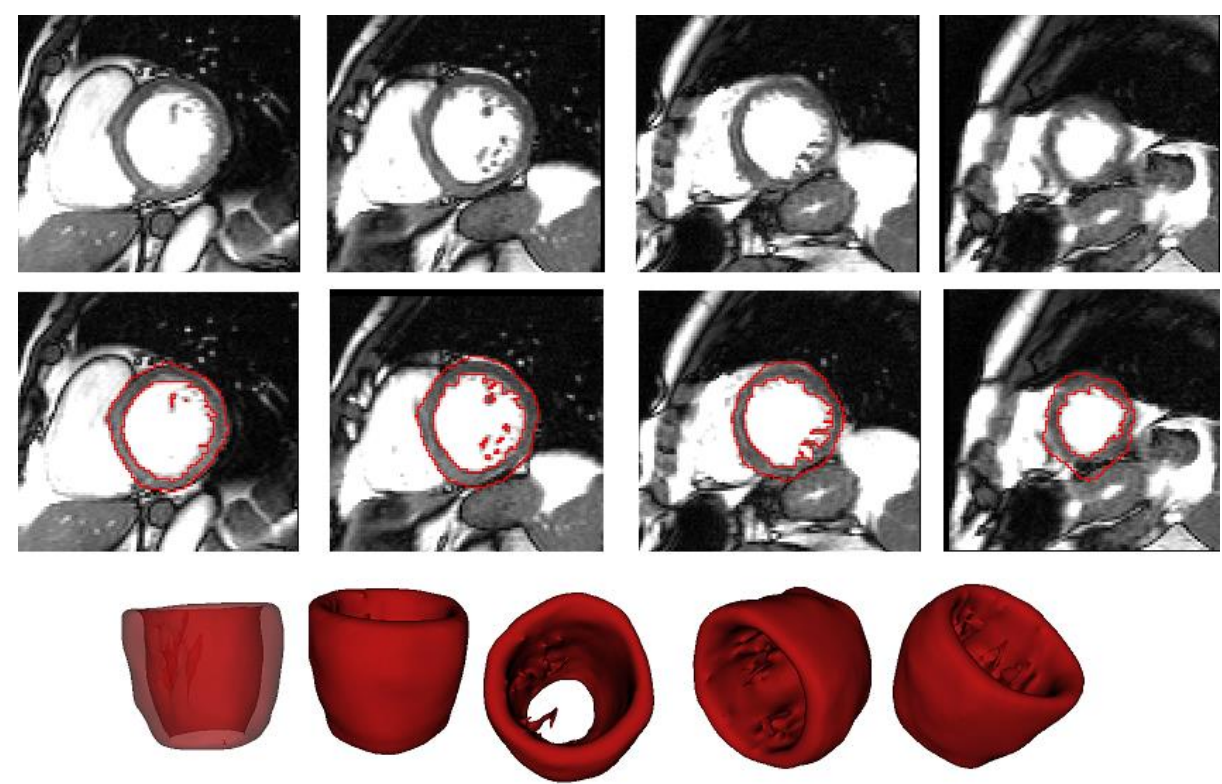

Fig.2.Illustration of the myocardium segmentation results: The first row shows the pseudo slices from apical to basal direction without any contours. The second row depicts the extraction of myocardial contours. The third row shows the extracted mesh from different corners.

\section{Conclusion}

The purpose of this work was to provide an automatic and accurate LV myocardial segmentation method for 3D cine-MRI images. Extraction of the LV endo- and epicardium has provided accurate and consistent results. Our Proposed method has achieved a mean Dice metric of 0.87 for the endocardium and 0.82 for the epicardium. Therefore, we focused on the volumetric object reconstruction (3D visualization) by exporting the endocardial and the epicardial extracted contours as 3D surface meshes [25]. This reconstruction is ensured throughout a complete cardiac cycle (from systole to diastole) to allow dynamic visualization of the LV. 


\section{References}

1. Olimulder, M. A. G. M., J. Van Es, and M. A. Galjee. "The importance of cardiac MRI as a diagnostic tool in viral myocarditis-induced cardiomyopathy." Netherlands Heart Journal17.12 (2009): 481-486.

2. Buser, Peter T., et al. "Noninvasive evaluation of global left ventricular function with use of cine nuclear magnetic resonance." Journal of the American College of Cardiology 13.6 (1989): 1294-1300.

3. Ginat, Daniel T., et al. "Cardiac imaging: Part 1, MR pulse sequences, imaging planes, and basic anatomy." American Journal of Roentgenology 197.4 (2011): 808-815.

4. Petitjean, Caroline, and Jean-Nicolas Dacher. "A review of segmentation methods in short axis cardiac MR images." Medical image analysis 15.2 (2011): 169-184.

5. Katouzian, Amin, Ashwin Prakash, and Elisa Konofagou. "A new automated technique for left-and right-ventricular segmentation in magnetic resonance imaging." 2006 International Conference of the IEEE Engineering in Medicine and Biology Society. IEEE, 2006.

6. Jolly, M. "Fully automatic left ventricle segmentation in cardiac cine MR images using registration and minimum surfaces." The MIDAS Journal-Cardiac MR Left Ventricle Segmentation Challenge 4 (2009): 59.

7. Lin, Xiang, Brett R. Cowan, and Alistair A. Young. "Automated detection of left ventricle in 4D MR images: experience from a large study." International Conference on Medical Image Computing and Computer-Assisted Intervention. Springer, Berlin, Heidelberg, 2006.

8. Pednekar, Amol, et al. "Automated left ventricular segmentation in cardiac MRI." IEEE Transactions on Biomedical Engineering53.7 (2006): 1425-1428.

9. Lorenzo-Valdés, Maria, et al. "Segmentation of 4D cardiac MR images using a probabilistic atlas and the EM algorithm." Medical Image Analysis 8.3 (2004): 255-265.

10. Pennell, Dudley J., et al. "Clinical indications for cardiovascular magnetic resonance (CMR): Consensus Panel report." Journal of Cardiovascular Magnetic Resonance 6.4 (2004): 727-765.

11. Mitchell, Steven C., et al. "3-D active appearance models: segmentation of cardiac MR and ultrasound images." IEEE transactions on medical imaging 21.9 (2002): 1167-1178.

12. Van Assen, Hans C., et al. "SPASM: a 3D-ASM for segmentation of sparse and arbitrarily oriented cardiac MRI data." Medical Image Analysis 10.2 (2006): 286-303.

13. Zhuang, Xiahai, et al. "Robust registration between cardiac MRI images and atlas for segmentation propagation." Medical Imaging 2008: Image Processing. Vol. 6914. International Society for Optics and Photonics, 2008.

14. Lötjönen, Jyrki, et al. "Statistical shape model of atria, ventricles and epicardium from short-and long-axis MR images." Medical image analysis 8.3 (2004): 371-386.

15. Beucher, Serge. "Use of watersheds in contour detection." Proceedings of the International Workshop on Image Processing. CCETT, 1979.

16. Mahmoudi, Ramzi, Mohamed Akil, and Mohamed HédiBedoui. "Concurrent computation of topological watershed on shared memory parallel machines." Parallel Computing 69 (2017): 78-97.

17. Soille, Pierre. "Morphological image analysis applied to crop field mapping." Image and Vision computing 18.13 (2000): 1025-1032.

18. Couprie, Michel, and Gilles Bertrand. "Topology preserving alternating sequential filter for smoothing two-dimensional and three-dimensional objects." Journal of Electronic Imaging 13.4 (2004): 720-731. 
19. Udupa, Jayaram K., et al. "A framework for evaluating image segmentation algorithms." Computerized medical imaging and graphics 30.2 (2006): 75-87.

20. Kaus, Michael R., et al. "Automated segmentation of the left ventricle in cardiac MRI." Medical image analysis 8.3 (2004): 245-254.

21. Lu, Ying-Li, et al. "Automatic functional analysis of left ventricle in cardiac cine MRI." Quantitative imaging in medicine and surgery 3.4 (2013): 200.

22. Hu, Huaifei, et al. "Automatic segmentation of the left ventricle in cardiac MRI using local binary fitting model and dynamic programming techniques." PloS one 9.12 (2014): e114760.

23. Irshad, Mehreen, et al. "Automatic segmentation of the left ventricle in a cardiac MR short axis image using blind morphological operation." The European Physical Journal Plus133.4 (2018): 148.

24. Lachaud, Jacques-Olivier, and Annick Montanvert. "Deformable meshes with automated topology changes for coarse-to-fine three-dimensional surface extraction." Medical Image Analysis3.2 (1999): 187-207.

25. Lorensen, William E., and Harvey E. Cline. "Marching cubes: A high resolution 3D surface construction algorithm." ACM siggraph computer graphics. Vol. 21. No. 4. ACM, 1987.

26. Vollmer, Jörg, Robert Mencl, and Heinrich Mueller. "Improved laplacian smoothing of noisy surface meshes." Computer graphics forum. Vol. 18. No. 3. Oxford, UK and Boston, USA: Blackwell Publishers Ltd, 1999.

27. Wesarg, Stefan. "AHA conform analysis of myocardial function using and extending the toolkits ITK and VTK." International Congress Series. Vol. 1281. Elsevier, 2005. 